\title{
A celebration of the mathematical work of Glenn Stevens
}

\section{Citation}

Mazur, Barry. 2016. "A Celebration of the Mathematical Work of Glenn Stevens." Ann. Math. Québec 40 (1) (January 8): 1-16. doi:10.1007/s40316-015-0053-3.

\section{Published Version}

doi:10.1007/s40316-015-0053-3

\section{Permanent link}

http://nrs.harvard.edu/urn-3:HUL.InstRepos:30403698

\section{Terms of Use}

This article was downloaded from Harvard University's DASH repository, and is made available under the terms and conditions applicable to Open Access Policy Articles, as set forth at http:// nrs.harvard.edu/urn-3:HUL.InstRepos:dash.current.terms-of-use\#OAP

\section{Share Your Story}

The Harvard community has made this article openly available.

Please share how this access benefits you. Submit a story.

Accessibility 


\title{
A celebration of the mathematical work of Glenn Stevens
}

\author{
Barry Mazur
}

June 10, 2015

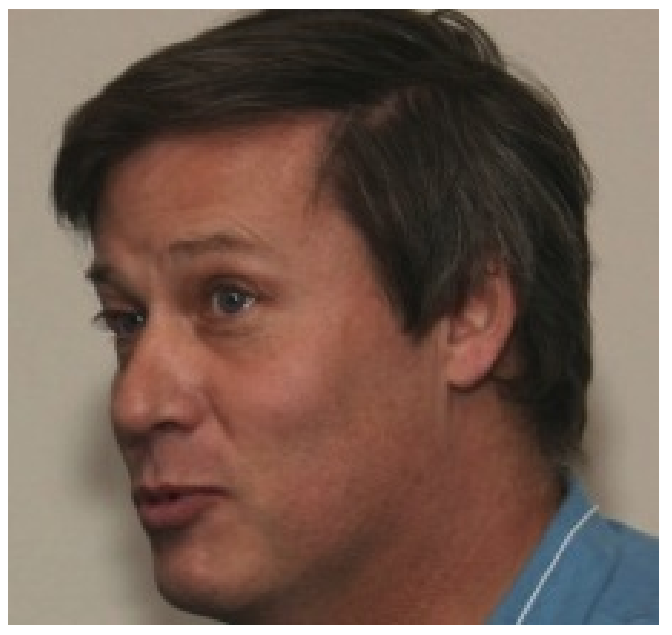




\section{Contents}

I Introduction $\quad 2$

1 The classical 'Arithmetic Questions' about elliptic curves 4

II Deformation, Overconvergence, and Euler Systems and their variants $\quad \mathbf{5}$

2 Rigid-analytic deformations 5

$\begin{array}{llr}3 & \text { Forms of Euler Systems } & 6\end{array}$

$\begin{array}{ll}\text { III } & \text { Interpolation } \\ \end{array}$

$\begin{array}{lll}4 & \text { The relevant interpolations over } \mathrm{C} & 7\end{array}$

$\begin{array}{lll}5 & \text { The parallel } p \text {-adic story } & 8\end{array}$

6 Nonparallelism $\quad 12$

$\begin{array}{ll}\text { IV Overconvergent Families of Modular Symbols } & 13\end{array}$

$\begin{array}{lll}7 & \text { Classical Modular Symbols } & 13\end{array}$

8 Rigid Analytic Modular Symbols $\quad 14$

$\begin{array}{ll}\mathrm{V} \text { The derivative of the } U_{p} \text {-eigenvalue with respect to weight } & 18\end{array}$ 


\section{Part I}

\section{Introduction}

This volume stems from the Conference in Honor of Glenn Stevens, held on June 2, 2014, at Boston University. What a pleasure it is to think about the excellent contributions Glenn has made so far in his - what I think of from my vantage point - still young life, and to see how it inspires and folds into the many current projects of the grand subject-p-adic variation in automorphic forms and in the wider context of arithmetic.

Glenn was a student of mine, and already as a graduate student had such firm drive, direction, and maturity that - when we went running together around Fresh Pond in Cambridge, chatting about mathematics, I would often feel I learned vastly more than I was teaching. His thesis and first book has served as source-works for me as soon as he wrote them. His grand contributions - so important for our subject - start from stunningly simple ideas. One of Glenn's constructions, the subject of Part IV of these notes, begins with the extremely original opening move ${ }^{1}$ of embedding the module of polynomials of degree $\leq k$ into a power series ring. And from there, it takes off!

Glenn has also been the architect and energetic force behind the astonishingly effective Promys program, an institution that has enriched our subject, mathematics, in ways that other intellectual disciplines must envy. That young people - enthusiastic learners and counselors - be introduced to both the subject and to each other, strengthening bonds of friendship to shape the eventual intellectual community in the marvelous way that our mathematical community enjoys, is of extreme importance. And the tack taken in the day-to-day work of Promys just radiates with such pragmatic optimism and joy: "prove it, disprove it, or deal with it," being one of the mottoes.

Some of the things Glenn's been quoted as having said to students, I try to take as guides for my own teaching. Glenn has a knack of crisply pointing out to students ways of navigating within our subject, and of achieving a deep appreciation of what it means to do mathematics. Here is an example of such a (seemingly simple) piece of advice, but one that gets to the heart of things: a student came to Glenn, swamped by perplexity when thinking about a certain problem in mathematics, and Glenn pressed the point:

It is good to be confused!

I think it is important to tell students this: that confusion is a tool, a useful goad, an unavoidable aspect of our work, and yet: it can be flipped from being an irritant to a deep source of enjoyment ${ }^{2}$.

\footnotetext{
${ }^{1}$ as Kevin Buzzard reflected in the banquet of the conference in Glenn's honor.

${ }^{2}$ I don't mean this to deflect blame from me, though, if this lecture turns out to be confusing!
} 


\section{The classical 'Arithmetic Questions' about elliptic curves}

Here is a basic list of arithmetic issues that we tend to ask about, when we study elliptic curves over number fields.

- Periods,

- Mordell-Weil groups endowed with their Néron-Tate heights; the corresponding regulators,

- Shafarevich-Tate groups,

- (Related asymptotics:)

- Frobenius distributions,

- Variation over 'twist families.'

We often have these tools to make a two-pronged attack:

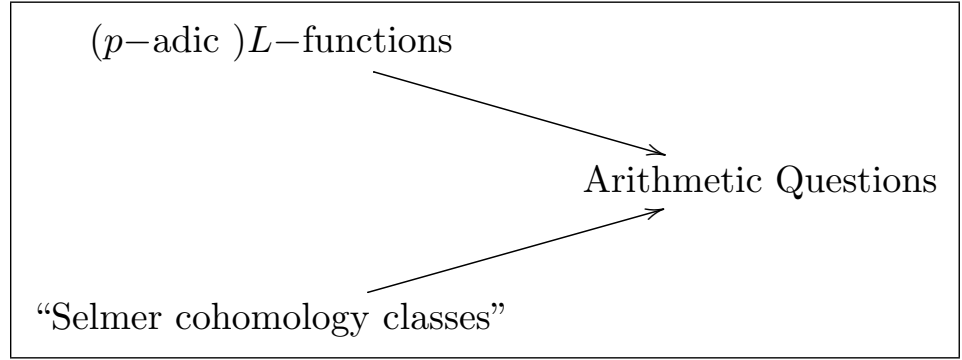

At times, a more extensive toolkit is available:

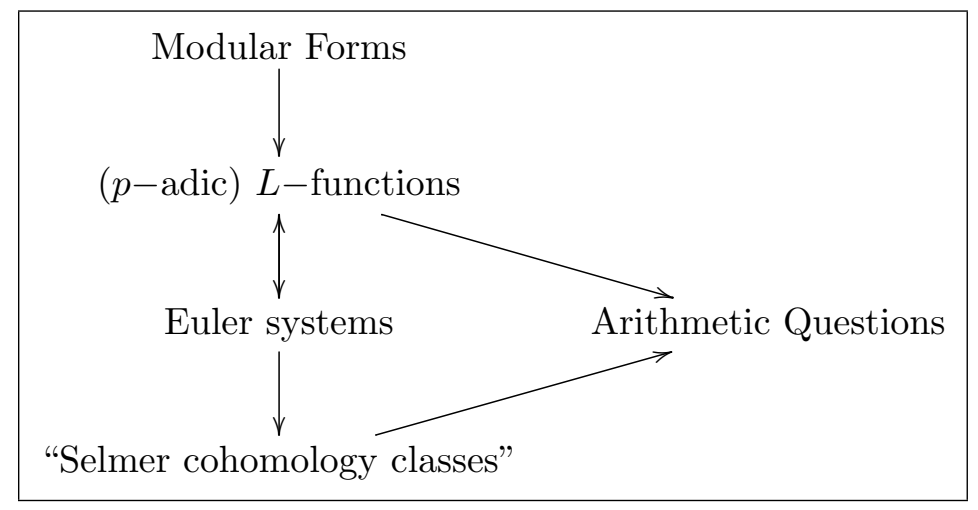

But what about variation? For example, if we are dealing with an elliptic curve $E$ over the rational number field, we can replace $E$ by its uniformizing modular eigenform, $f_{E}$ and note that $f_{E}$ lives in the eigencurve, a one-parameter $p$-adic analytic family of $p$-adic (overconvergent) Hecke eigenforms, 
each of these eigenforms raising 'Arithmetic Questions' somewhat similar to the questions raised by the elliptic curve $E$. The picture of the above chart, when available, gets even more interesting when you consider its variation over the eigencurve.

\section{Part II}

\section{Deformation, Overconvergence, and Euler Systems and their variants}

The concepts in the title above, and in the chart below, represent some intensely pursued programs in number theory, and will be themes well-covered in this volume. They connect to basic constructions and arithmetic questions through the intermediary $p$-adically varying families:

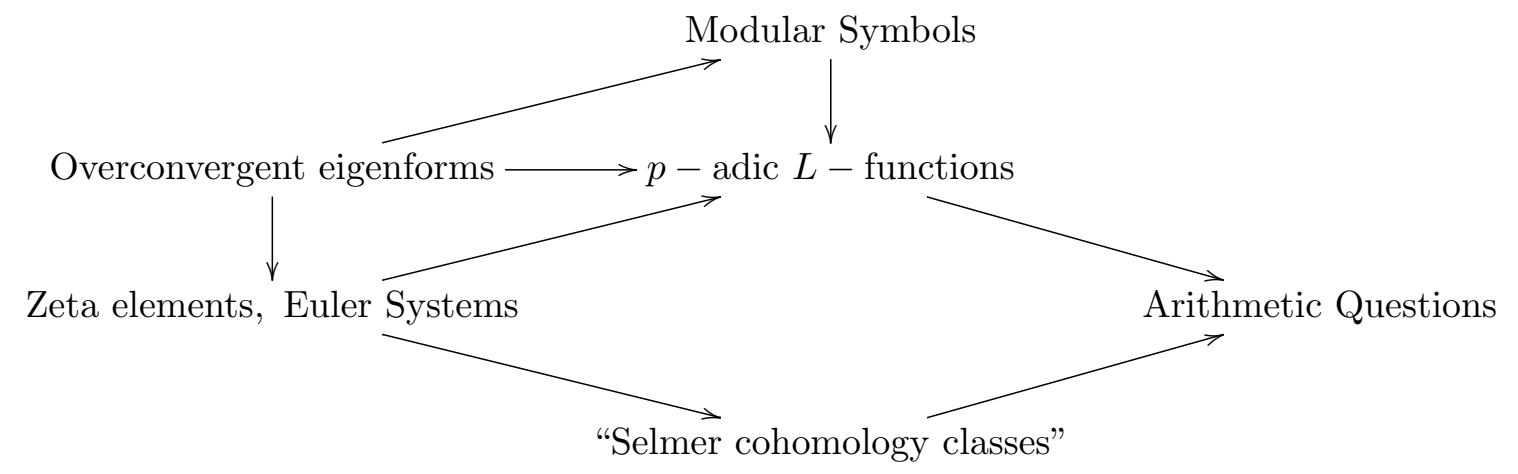

Let's start with an overview by first merely listing some of the ways these concepts show up - both in Glenn's work and in what will appear in this volume. Once this is done, I'll back up and present a more introductory view of some of these structures.

\section{$2 \quad$ Rigid-analytic deformations}

One challenge to our subject is to establish $p$-adic rigid analytic families of the constellation of structures that form the chart above. That is we might aim for rigid analytic variations

- of overconvergent modular and automorphic forms (organized in eigenvarieties) and

- of the $p$-adic $L$ functions of these modular and automorphic forms (organized as functions on the corresponding eigenvarieties)

- of their associated modular symbols; specifically, of Glenn's theory of families of overconvergent modular symbols and more generally of overconvergent cohomology of arithmetic subgroups of reductive groups with coefficients in certain modules of distributions. 
- of "zeta-elements," and whatever generalized Euler systems of various "core ranks" are allowed, in each specific context.

Glenn's work has been fundamental for all of these topics over decades. For example, the notes for his IHP (2000) course on families of overconvergent modular symbols has been instrumental in providing a fluid approach to many of these ideas. His breakthrough, a decade earlier, with Ralph Greenberg - expressing $\mathcal{L}$-invariants as the derivative (with respect to weight) of the eigenvalue of Atkin-Lehner's operator $U_{p}$-opened up a still-vital chapter of our subject. His more recent construction (2013) with Rob Pollack of $p$-adic $L$-functions associated to overconvergent eigenforms at critical slopes is both beautiful and revelatory of subtle issues in the theory of eigencurves. An impressive list of pdf files of published work, and yet-to-be-published preprints, of Glenn and collaborators on these issues can be found in http://math.bu.edu/people/ghs/research.html.

Much of this volume involves exactly these themes, with on-going constructions in new important directions, of eigenvarieties ${ }^{3}$ as parameter spaces for: finite slope families of overconvergent automorphic eigenforms and their corresponding families of modular symbols ${ }^{4}$, Galois representations ${ }^{5}$ $L$-functions and/or their $\zeta$-elements, Euler systems, and regulators ${ }^{6}$.

We will also see an intriguing differential aspect (in the form of a DGA algebra structure related of Hecke algebras as one climbs $p$-powers in level $)^{7}$, an application of the work of Ash and Stevens in the computation of certain periods on rigid analytic spaces related to modular forms ${ }^{8}$, and a technique, via averaging, for guaranteeing the nonvanishing of certain moments of $L$-function values ${ }^{9}$.

\section{$3 \quad$ Forms of Euler Systems}

One exciting phenomenon that seems to be emerging, and is visible in the subjects of a few of the articles in this volume, is the evolution of what one might call "forms of Euler systems." The basic template for Euler Systems had been given in the fundamental work of Kolyvagin [3]. In Karl Rubin's book Euler Systems [6] it was formatted in an extremely efficient way that encompassed circular- and elliptic-unit Euler systems, as well as those coming from Heegner points and higher dimensional cycle constructions, or from Kato's construction using Beilinson classes (cf. Tony Scholl's [7]). Other formats appeared in the work of Matheus Flach [2] (or the "coherent Flach systems" in [4] and Weston [9]) which didn't have the full range of the original Kolyvagin-type collection of cohomology classes in large quantities of abelian extensions of the base field. We see emerging even more new configurations of cohomology classes that control arithmetic behavior, among which are interpolation over $p$-adic parameters, and conjectured 'higher core rank' systems-

\footnotetext{
${ }^{3}$ as in the talks of Avner Ash, Joel Bellaiche, Ellen Eischen, Michael Harris and Eugene Hellman

${ }^{4}$ as in the talks of Fabrizio Andreatta, and Ander Steele

${ }^{5}$ as in the talks of Benoit Stroh and Jacques Tilouine

${ }^{6}$ as in the talks of Francesc Castella, Masataka Chida, David Hansen, Masato Kurihara, David Loeffler, Jan Nekovar, Viktor Rotger, Eric Urban, and Sarah Zerbes

${ }^{7}$ in the talk of Jeehon Park

${ }^{8}$ in the talk of Marco Seveso

${ }^{9}$ in the talk of Jeehon Park
} 
i.e., classes in wedge-powers of cohomology groups as had already been envisioned in the work of Perrin-Riou.

\section{Part III}

\section{Interpolation}

A fundamental way of constructing $p$-adic variation, or $p$-adic deformation, is via $p$-adic interpolation. 'Interpolation' itself has a long (and mostly unwritten, I think) history.

\section{The relevant interpolations over $\mathrm{C}$}

Interpolation has many aspects, but for us, the great classical moments that precede our $p$-adic subject comes from these acts of interpolation.

1. Exponentiation: $f_{n}(k):=n^{k}$; for $k \in \mathbf{Z} \quad-----\rightarrow f_{n}(s):=n^{s}=e^{s \cdot \log n}$ for $s \in \mathbf{C}$.

2. Analytic Continuation: Euler's $\zeta(k)=\sum_{n=1}^{\infty} n^{-k}$ (for $k \in \mathbf{Z}, k \geq 2$ ) to Riemann's zetafunction $\zeta(s)$ (for $s \in \mathbf{C}-\{1\}$ ).

This passage, $\zeta(k)(k \geq 2)----\rightarrow \zeta(s)(s \neq 1)$, can be viewed as an honest interpolation in various ways. For example,

- A theorem of Deninger [1] tells us that-given the known growth properties of the complex analytic function $\zeta(s)$ arising from some Dirichlet series - such a function is uniquely determined by its integral values - and even by the values: $k \mapsto \zeta(k)$ for $k \geq k_{0}$ (for any $k_{0}>1$ ).

- A theorem of Hasse simply gives $\zeta(s)$ as a limit of this convergent series:

$$
\zeta(s)=\frac{1}{s-1} \sum_{n=0}^{\infty} \frac{1}{n+1} \sum_{k=0}^{n}\left(\begin{array}{l}
n \\
k
\end{array}\right) \frac{(-1)^{k}}{(k+1)^{s-1}} .
$$

\section{Variation of automorphic forms parametrized by weight}

Consider the interpolation of Eisenstein series at classical integral weights $s=3,4,5, \ldots$ to provide either the non-analytic parametrized family of Eisenstein series

$$
\operatorname{Eis}_{s}^{\{\infty\}}(z):=\frac{1}{2} \sum_{g c d(m, n)=1} \frac{y^{s}}{|m z+n|^{2 s}}
$$


for $z=x+i y$ in the upper half plane $y>0$. that had beginnings in work of Hecke and Reidemeister, and was developed by Maass (and, of course, later much more substantially developed by Langlands). Or, perhaps more directly germane to us, consider:

$$
\mathcal{E}_{s}(q):=\frac{1}{2} \zeta(1-s)+\sum_{n=1}^{\infty}\left\{\sum_{d \mid n} d^{s-1}\right\} q^{n}
$$

which fits into the following framework:

Let $\Lambda_{\infty}$ be the ring of complex analytic functions expressible as Dirichlet series in appropriate half-planes that extend to complex analytic functions on the entire plane, bounded in vertical strips. Then

$$
\mathcal{E}_{s}(q) \in \Lambda_{\infty}[[q]]
$$

and has the property that its specializations to $s=2,4,6, \ldots, 2 k, \ldots$ yield the classical Eisenstein series of level 1 at those weights.

\section{$5 \quad$ The parallel $p$-adic story}

We'll exclude the case $p=2$ from now on.

1. Exponentiation: Fermat's Little Theorem as expanded by Euler gives the congruence we'lll call Euler's Theorem:

$$
n^{k^{\prime}} \equiv n^{k} \quad \bmod p^{r}
$$

for any $n £ 0 \quad \bmod p$ and pair of exponents $k^{\prime} \equiv k \bmod (p-1) p^{r-1}$.

It is natural to pass to the (projective) limit of the sequence:

$$
\cdots \rightarrow \mathbf{Z} /(p-1) p^{r-1} \mathbf{Z} \rightarrow \cdots \rightarrow \mathbf{Z} /(p-1) p \mathbf{Z} \rightarrow \mathbf{Z} /(p-1) \mathbf{Z} .
$$

When the dust settles-i.e., as $r \mapsto \infty$-we get a continuous one-parameter $p$-adic space (a commutative Lie group, in fact)

$$
W:=\lim _{r \rightarrow \infty} \mathbf{Z} / \phi\left(p^{r}\right) \mathbf{Z}=\lim _{r \rightarrow \infty} \mathbf{Z} /\left(p^{r-1}(p-1) \mathbf{Z}\right)=\lim _{r \rightarrow \infty} \mathbf{Z} / p^{r-1} \mathbf{Z} \times \mathbf{Z} /(p-1) \mathbf{Z}
$$

which we will refer to as $p$-adic weight space. This isomorphism provides $W$ with a canonical product decomposition

$$
W=\mathbf{Z}_{p} \times \mathbf{Z} /(p-1) \mathbf{Z}
$$

and we'll write $\kappa=(s, i)$ following this product decomposition, with $s \in \mathbf{Z}_{p}$, and $i \in \mathbf{Z} /(p-1) \mathbf{Z}$ being the image of $\kappa$ under the projections to the factors. Any element $\kappa \in W$ can be viewed as 'being' a locally analytic character $\kappa: \mathbf{Z}_{p}^{*} \rightarrow \mathbf{Z}_{p}^{*}$ by continuity (i.e., approximate $\kappa$ by an 
integer $k$ modulo $(p-1) p^{N-1}$ and note that raising to the $k$-th power yields a sequence of characters that converges as $k \rightarrow \kappa)$.

Viewing $W$ geometrically, it is a union of $p-1$ disjoint closed unit discs:

$$
W=\sqcup_{i \in \mathbf{Z} /(p-1) \mathbf{Z}} W_{i}
$$

where $W_{i}$ is the inverse image of $i$ under the natural map $W \rightarrow \mathbf{Z} /(p-1) \mathbf{Z}$. $W$ contains the monoid of natural numbers

$$
\mathbf{N} \subset W
$$

the elements of which are called "classical weights." Since $p$ is assumed odd, there is a natural further projection:

$$
W \rightarrow \mathbf{Z} /(p-1) \mathbf{Z} \rightarrow \mathbf{Z} / 2 \mathbf{Z}
$$

allowing us to decompose $W$ into even weights and odd weights; this will be useful below.

$$
W=W_{\text {even }} \sqcup W_{\text {odd }}
$$

For each point $\kappa \in W$ and any integer $d$, we can define

$$
d^{\{\kappa\}}:=\lim _{r \rightarrow \infty} d^{w_{r}} \in \lim _{r \rightarrow \infty} \mathbf{Z} / p^{r} \mathbf{Z} \simeq \mathbf{Z}_{p} .
$$

where the sequence $\left\{w_{r}\right\}_{r}$ are positive integers tending to infinity ${ }^{10}$ such that $w_{r} \equiv w$ $\bmod \phi\left(p^{r}\right) \mathbf{Z}$ for all $r$. Note that if $d$ is divisible by $p$ then $d^{\{\kappa\}}=0$.

\section{2. $p$-Adic Analytic Continuation}

For $\kappa \in W_{\text {even }}$, the important construction of Kubota and Leopoldt-i.e., their " $p$-adic $L$ function"- performs, in effect, an analogous interpolation of the Riemann zeta-function and Dirichlet $L$-functions. Appropriately normalized, the values of these functions at odd negative integers, is packaged by the function

$$
k \mapsto-b_{k} / 2 k \in \mathbf{Q}
$$

where $k$ is the $k$-th Bernoulli number. A consequence of the classical study of Bernoulli numbers (The Von Staudt-Clausen Theorem along with the Kummer congruences) is, first, that for positive integers $k \in W_{\text {even }}-W_{0}$ we may view $-b_{k} / 2 k \in \mathbf{Z}_{p}$ and then, fixing a sequence of even positive integers $\left\{k_{j}\right\}_{j}$ which go to infinity (when viewed in $\mathbf{R}$ ) and which have the limit

$$
\lim _{j \rightarrow \infty} k_{j}=\kappa \in W_{\text {even }}-W_{0}
$$

(when viewed in $W$ ) we obtain the convergent limit

$$
-b_{\kappa} / 2 \kappa:=\lim _{j}-b_{k_{j}} / 2 k_{j} \in \mathbf{Z}_{p}
$$

\footnotetext{
${ }^{10}$ Note that even if $w$ is an ordinary integer, we will want the approximating $w_{r}$ 's to be positive numbers tending to infinity.
} 
thereby interpolating the function $k \mapsto-b_{k} / 2 k$ to a $p$-adic analytic function on $W_{\text {even }}-W_{0}$ :

$$
\kappa \mapsto-b_{\kappa} / 2 \kappa:=\lim _{j}-b_{k_{j}} / 2 k_{j} \in \mathbf{Z}_{p}
$$

Writing $\kappa=(s, i)$ as discussed above, one defines the Kubota-Leopoldt $p$-adic $L$-function:

$$
L_{p}\left(1-s, \omega^{i}\right):=-b_{\kappa} / \kappa
$$

Here $\omega$ designates the Teichmüller character.

\section{Variation of automorphic forms parametrized by weight}

The power of Fermat's Little Theorem to generate congruences is illustrated by comparing the classical Eisenstein series of weight $k$ (here $k \geq 2, k$ is even; and keep $k \neq \equiv 0 \bmod (p-1)$ in this discussion).

$$
G_{k}=-\frac{b_{k}}{2 k}+\sum_{n=1}^{\infty}\left\{\sum_{d \mid n} d^{k-1}\right\} q^{n}
$$

taken modulo $p$ (i.e., its Fourier coefficients being taken modulo $p$ ) with the Eisenstein series of weight $k+p-1$

$$
G_{k+p-1}=-\frac{b_{k+p-1}}{2(k+p-1)}+\sum_{n=1}^{\infty}\left\{\sum_{d \mid n} d^{k-1} d^{p-1}\right\} q^{n}
$$

or, more generally, comparing it modulo $p^{r}$ with the Eisenstein series of weight $k+\phi\left(p^{r}\right)$ :

$$
G_{k+\phi\left(p^{r}\right)}=-\frac{b_{k+\phi\left(p^{r}\right)}}{2\left(k+\phi\left(p^{r}\right)\right)}+\sum_{n=1}^{\infty}\left\{\sum_{d \mid n} d^{k-1+\phi\left(p^{r}\right)}\right\} q^{n} .
$$

Here it is Euler's Theorem that guarantees that the nonconstant coefficients of $G_{k+\phi\left(p^{r}\right)}$ are congruent to the corresponding nonconstant coefficients of $G_{k}$ modulo $p^{r}$. And it is the classical Kummer congruence that guarantees the analogous result for the constant coefficients, giving us that

$$
G_{k+\phi\left(p^{r}\right)} \equiv G_{k} \quad \bmod p^{r} .
$$

In fact, an argument of Serre developed by Katz allows you to use modularity of these Fourier series together with the congruences we've just discussed between nonconstant coefficients to prove the analogous congruence for the constant coefficients; i.e., to prove the classical Kummer congruences as a derivative of, in effect, Euler's Theorem.

Putting all this together we have a construction of Serre ${ }^{11}$ : a $p$-adic interpolation of the Fourier coefficients of the classical family of Eisenstein series. More exactly we may form, for every weight $\kappa \in W_{\text {even }}$ such that $\kappa$ projects to the even number $i \neq 0$ modulo $p-1$, the $p$-adic continuous series of ' $p$-adic Hecke eigenforms on $\Gamma_{1}(p)$ ':

\footnotetext{
${ }^{11}$ in [8]: Formes modulaires et fonctions zêta $p$-adiques
} 


$$
G_{\kappa}^{\{p\}}(q):=-\frac{1}{2} L_{p}\left(1-s ; \omega^{i}\right)+\sum_{n}\left\{\sum_{d \mid n} d^{\{\kappa\}-1}\right\} \cdot q^{n} \subset \mathbf{Z}_{p}[[q]]
$$

The half-scare quotes around 'p-adic Hecke eigenforms on $\Gamma_{0}(p)$ ' are just to record that one has to give a sense of what this means, specifically for nonclassical weights $\kappa$ : these are $p$-adic overconvergent eigenforms. But even when $\kappa$ is the image of an even integer $k \geq 2$, and even though, in this case, $G_{\kappa}^{\{p\}}(q)$ is identifiable with a classical eigenform, there is a significant difference between $G_{k}(q)$ and $G_{\kappa}^{\{p\}}(q)$. For one thing, the coefficients of $G_{\kappa}^{\{p\}}(q)$ are just $p$-adic numbers; however they are in the image of

$$
\mathbf{Q}\left(\mu_{p-1}\right) \hookrightarrow \mathbf{Q}_{p}
$$

where we make an identification

$$
\mu_{p-1} \stackrel{\sigma}{\simeq} \mathbf{F}_{p}^{*}
$$

and then embed

$$
\mathbf{F}_{p}^{*} \subset \mathbf{Z}_{p}^{*} \subset \mathbf{Q}_{p}^{*} \subset \mathbf{Q}_{p}
$$

in the natural way. Using such an identification we may view $G_{\kappa}^{\{p\}}(q)$ as a genuine classical modular form with Fourier coefficients in $\mathbf{Q}\left(\mu_{p-1}\right) \hookrightarrow \mathbf{C}$; it has weight $k$ and is on the group $\Gamma_{0}(p)$ with a nebentypus character that depends on $\sigma$ and $k$.

Also, we don't just throw away the Eisenstein series with weights $2 \leq k \equiv 0 \bmod p-1$, these having - by the von Staudt-Clausen Theorem - a constant term $-\frac{b_{k}}{2 k}$ with negative ord ${ }_{p}$. We have other plans for these Eisenstein series: We just divide by their constant terms to get a sequence

$$
E_{k}(q)=1-\frac{2 k}{b_{k}} \sum_{n=1}^{\infty}\left\{\sum_{d \mid n} d^{k-1}\right\} q^{n}
$$

which has the very useful property of being $\equiv 1 \bmod p$, and which interpolates to give another family

$$
E_{\kappa}^{\{p\}}(q)
$$

for $\kappa \in W_{0} \subset W_{\text {even }}$. The Fourier expansion of any member of this family is congruent to 1 $\bmod p$, and if $\kappa=0 \in W_{0}$ then

$$
E_{\kappa}^{\{p\}}(q)=E_{0}^{\{p\}}(q)=1
$$

a fact that plays an important role in this story.

These families of eigenfunctions, varying $p$-adic analytically in their weights were first put forward by Serre, and can viewed as the starting point of a significant amount of modern ( $p$-adic) number theory. 


\section{$6 \quad$ Nonparallelism}

We have described three types of interpolation-exponentiation, zeta-function, Eisenstein series in the classical context (i.e., over the complex numbers) and the corresponding interpolations over the $p$-adic numbers. So far, we have discussed parallel developments in these contexts. But the $p$-adic context has further tools of analysis that is missing, at least at present, in the classical context.

The basic structures and properties that we encounter in the $p$-adic world of automorphic forms for which we don't quite see-or at least, don't yet see - corresponding concepts in the classical C-valued world of automorphic forms are:

- the distinction of ordinary and supersingular,

- the corresponding notion of overconvergence and-topping the list-

- the existence the Atkin-Lehner operation $U_{p}$, especially as its appearance as a compact operator on the $p$-adic Banach modules of overconvergent modular forms.

The $p$-adic modular eigenfunctions, and all the others we will be dealing with today all have the technical property of being overconvergent in the sense that

- they can be viewed 'geometrically' as sections of the appropriate line bundle on the appropriate $p$-adic modular curve,

- they are allowed to have essential singularities-but only of a specific controlled type - in small $p$-adic discs centered above the supersingular points in characteristic $p$.

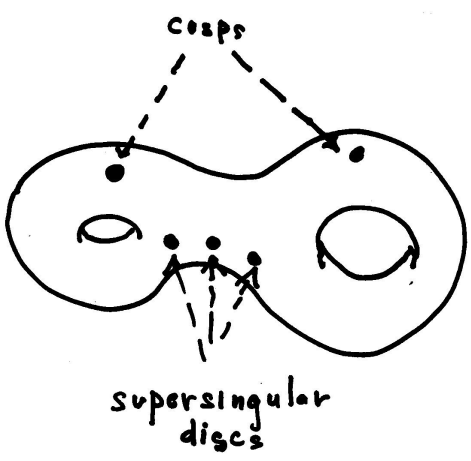


The Hecke operators and the $U_{p}$ operator act as correspondences on the modular curves and induce operators on the corresponding spaces of overconvergent sections. By "eigenform" we will always mean an overconvergent $p$-adic modular form that is an eigenvector for the appropriate Hecke operators. Since these sections may have (allowed) singularities at the supersingular points, one is dealing with noncompactness - when working with any of these families - whether or not one requires regularity at the cusps. This is the underlying reason why there can be continuous families of $p$-adic cuspidal eigenforms, i.e., despite the fact that the classical members of the family correspond to the discrete series in the (classical) harmonic analysis of these modular curves ${ }^{12}$.

\section{Part IV}

\section{Overconvergent Families of Modular Symbols}

\section{$7 \quad$ Classical Modular Symbols}

If $E$ is an elliptic curve over $\mathbf{Q}$ uniformized by the modular form $\omega_{E}$, with \pm -periods Per ${ }_{\mathrm{E}}^{ \pm}$given as the integrals of $\omega_{E}$ over the real and imaginary loci in $E(\mathbf{C})$, and $\tau_{E}:=$ the order of the torsion subgroup of $E(\mathbf{Q})$ then we have this chart where all the double-headed arrows mean what they indicate: you can determine (given $\operatorname{Per}_{\mathrm{E}}^{ \pm}$and $\tau_{E}$ ) the upper object from the lower and vice versa ${ }^{13}$.

\footnotetext{
${ }^{12}$ Perhaps the most succinct way of seeing an operation 'missing in the classical context' is to form the continuous families of 'modular forms' as follows. Multiply the continuous family of Eisenstein series (in the classical and in the p-adic context) by a classical cuspform (for example, $\Delta$, the modular form of weight 12 , and level 1 ) The family of modular forms you now have will be an interpolation of classical cusp forms in even weights $=2,4, \ldots$ but will no longer be eigenforms. The interpolated range of weights of your family will be, in the complex context, the entire complex plane; and will be $p$-adic weight space in the $p$-adic context. The natural impulse, then, would be to try to perform a spectral analysis of your family so as to achieve, at least for small ranges of weights, a decomposition of the family into a (presumably infinite) sum of families that are, in integral positive even weights, eigenforms. Thanks to the $U_{p}$ operator, this ispectral analysis can be done in the $p$-adic context, but is not (yet) do-able in the archimedean setting.

${ }^{13}$ The top double-headed arrow raises an interesting question, but the other two double-headed arrows are via simple formulae.
} 
Analytic issues; e.g., Regulators, etc.

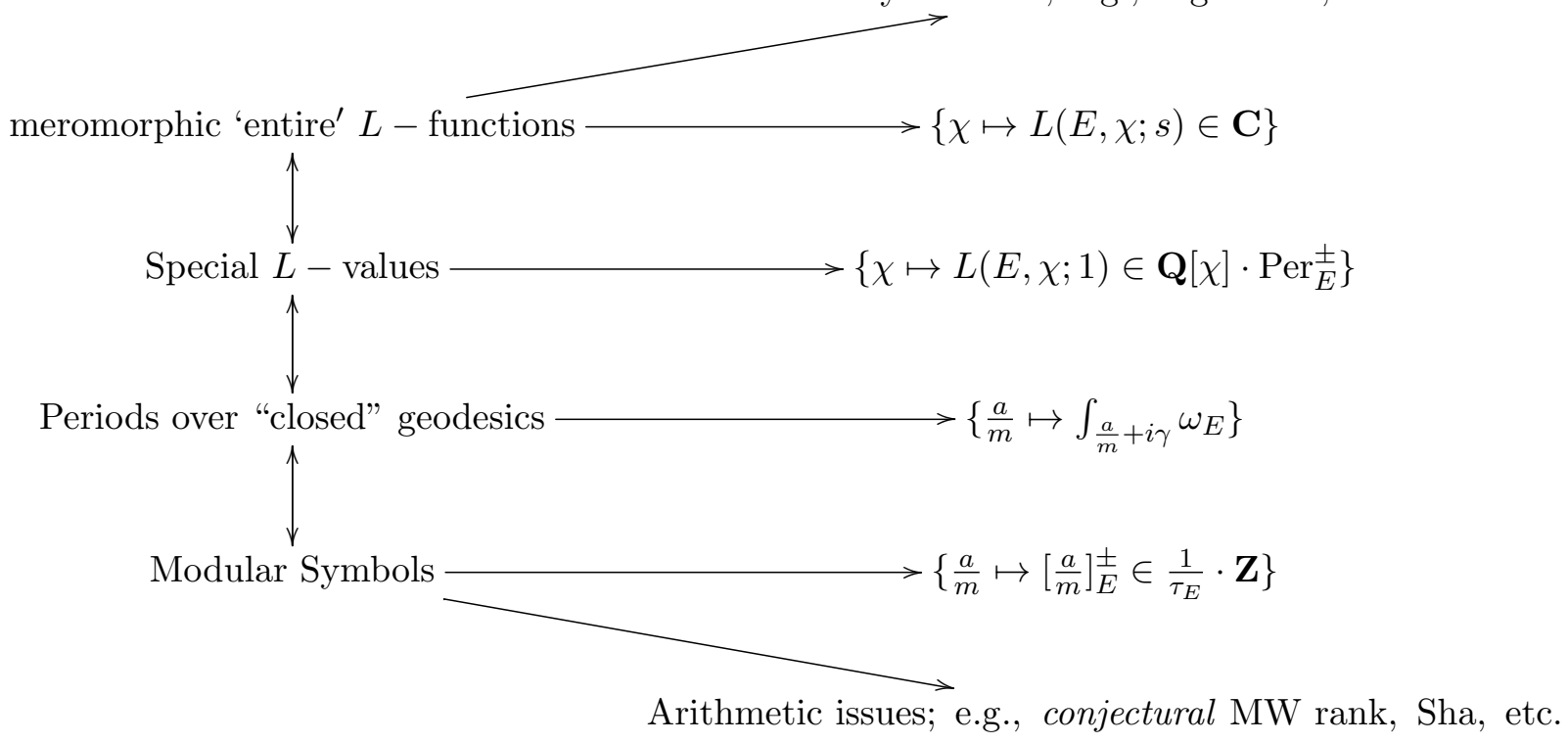

Extremely relevant for the control that one has over this structure is that the modular symbols $\frac{a}{m} \mapsto\left[\frac{a}{m}\right]_{E}^{ \pm}$viewed as functions on all of $\mathbf{Q} / \mathbf{Z}$ are determined, via an elementary algorithm-no more baroque that the Euclidean algorithm - from the finite set of its values

$$
a \mapsto\left[\frac{a}{N_{E}}\right]_{E}^{ \pm} \text {for } a=0,1,2, \ldots, N_{E}-1,
$$

where $N_{E}$ is the conductor of $E$.

The chart, wonderful though it is, is only for a single elliptic curve at a time. Taking an eigenvariety perspective: it is merely for a single point on the eigencurve!

\section{$8 \quad$ Rigid Analytic Modular Symbols}

The fundamental paper introducing this structure is Glenn Stevens' Rigid Analytic Modular Symbols (1994).

Let $p \geq 5$. As usual, $\mathbf{Q}_{p}[X]$ denotes the ring of polynomials in the variable $X$ with coefficients in $\mathbf{Q}_{p}$. For $k \geq 0$ let $P_{k}$ denote the (k+1-dimensional) $\mathbf{Q}_{p}$-vector subspace of $\mathbf{Q}_{p}[X]$ consisting of polynomials of degree $\leq k$. (Equivalently, you may homogenize these polynomials by introducing a variable $Y$ and view $P_{k}$ as the vector space of homogeneous polynomials of degree $k$ in $X, Y$.

The group $\mathrm{GL}_{2}\left(\mathbf{Q}_{p}\right)$ acts on $P_{k}$ as the $k$-th symmetric power of the standard representation on $P_{1}$ (i.e., via its linear fractional action on homogeneous linear polynomials in $X$ and $Y$. tThe action 
on the right via the transpose gives, for

$$
\gamma=\left(\begin{array}{ll}
a & b \\
c & d
\end{array}\right)
$$

the formula:

$$
(F \mid \gamma)(X, Y)=F(d X+b Y, c X+a Y)
$$

We have

$$
\Gamma_{0}(p) \subset \mathrm{GL}_{2}(\mathbf{Q}) \subset \mathrm{GL}_{2}\left(\mathbf{Q}_{p}\right)
$$

and can consider the $\mathbf{Q}_{p}$-linear action of $\Gamma_{0}(p)$ on $P_{k}$.

Definition 1. The space of classical modular symbols of weight $k$ is the compact onedimensional cohomology of $\Gamma_{0}(p)$ with values in $P_{k}$,

$$
\operatorname{Symb}\left(\Gamma_{0}(p) ; k\right):=H_{c}^{1}\left(\Gamma_{0}(p) ; P_{k}\right) .
$$

This terminology "symbol" comes from the alternate description of this cohomology group as given by equivariant functions $\phi$ from the left $\Gamma_{0}(p)$-module $\Delta$ of degree zero divisors on $P^{1}(\mathbf{Q})$ - the action being the natural linear fractional action-to the right $\Gamma_{0}(p)$-module $P_{k}$. Since

$$
\left(\begin{array}{ll}
1 & 1 \\
0 & 1
\end{array}\right) \in \Gamma_{0}(p)
$$

the equivariance property implies that $\phi([x]-[\infty])=\phi([x+n]-[\infty])$ for any $n \in \mathbf{Z}$, so these values are determined by the image of $x$ in $\mathbf{Q} / \mathbf{Z}$ and therefore, traditionally, modular symbols are often described as functions on $\mathbf{Q} / \mathbf{Z}$.

Since

$$
c:=\left(\begin{array}{cc}
-1 & 0 \\
0 & 1
\end{array}\right)
$$

normalized $\Gamma_{0}(p), c$ induces an involution on $\operatorname{Symb}\left(\Gamma_{0}(p) ; k\right)$, decomposing it "evenly," as

$$
\operatorname{Symb}\left(\Gamma_{0}(p) ; k\right)=\operatorname{Symb}\left(\Gamma_{0}(p) ; k\right)^{+} \oplus \operatorname{Symb}\left(\Gamma_{0}(p) ; k\right)^{-} .
$$

Note: The Eichler-Shimura correspondence relates the image of this cohomology module in the corresponding non-compact cohomology module with the space of cuspforms of level $\Gamma_{0}(p)$ and of weight $k+2$ (i.e., projects $\operatorname{Symb}\left(\Gamma_{0}(p) ; k\right)$ onto this space of cuspforms). The standard Hecke operators act on $H_{c}^{1}\left(\Gamma_{0}(p) ; P_{k}\right)$ compatibly with their action on modular forms of that weight and level.

Glenn's fundamental construction interpolates symbols, from

$$
k \mapsto \operatorname{Symb}\left(\Gamma_{0}(p) ; k\right) ; \quad k=0,1,2,3, \ldots
$$

to establish a continuum of $p$-adic Banach spaces, 


$$
\kappa \mapsto \operatorname{Symb}\left(\Gamma_{0}(p) ; \kappa\right)
$$

that are parametrized by ( $p$-adic) weight $\kappa$, thereby establishing a format for a construction of the eigencurve. The idea is elegant:

Start with the Tate algebra $\mathcal{A}:=\mathbf{Q}_{p}\langle x\rangle$ viewed as the Banach algebra over $\mathbf{Q}_{p}$ consisting of all formal power series $f(z)=\sum_{k=0}^{\infty} a_{k} x^{k} \in \mathbf{Q}_{p}[[x]]$ such that $\lim _{k \rightarrow \infty} a_{k}=0$ equipped with Banach norm $\|f\|$ given as the sup of the absolute value of its coefficients. Let $\mathcal{D}$ denote the Banach space of $\mathbf{Q}_{p}$-valued continuous functionals on $\mathcal{A}$.

So we have a continuous bilinear pairing: $(\mu, f) \mapsto\langle\mu, f\rangle$ for $\mu \in \mathcal{D}$ and $f \in \mathcal{A}$.

Let $\Sigma_{0}(p)$ denote the semigroup of nonsingular matrices in $\operatorname{Mat}_{2}\left(\mathbf{Z}_{p}\right)$

$$
\gamma=\left(\begin{array}{ll}
a & b \\
c & d
\end{array}\right)
$$

with $a \in \mathbf{Z}_{p}^{*}$, and $c \in p \mathbf{Z}_{p}$. We have the natural inclusion,

$$
\Gamma_{0}(p) \subset \Sigma_{0}(p)
$$

Moreover, for any locally analytic character $\kappa: \mathbf{Z}_{p}^{*} \rightarrow \mathbf{Z}_{p}^{*}$ we have the (continuous) weight $\kappa$-action of the semigroup $\Sigma_{0}(p)$ on $\mathcal{A}$ given by the formula:

$$
\left(\gamma_{\kappa} f\right)(x)=\kappa(a+c x) \cdot f\left(\frac{b+d x}{a+c x}\right) .
$$

Define the induced (continuous) weight $\kappa$-action of $\Sigma_{0}(p)$ on $\mathcal{D}$ by the (dual) formula

$$
\left\langle\mu \gamma_{\kappa}, f\right\rangle=\left\langle\mu, \gamma_{\kappa} f\right\rangle
$$

Denote by $\mathcal{D}_{\kappa}$ the $\mathbf{Q}_{p^{-}}$Banach space $\mathcal{D}$ equipped with the weight $\kappa$-action of $\Sigma_{0}(p)$.

Definition 2. (Glenn) The space of rigid analytic modular symbols of weight $\kappa$ is the compact one-dimensional cohomology of $\Gamma_{0}(p)$ with values in the $\mathbf{Q}_{p}$-Banach space $\mathcal{D}_{\kappa}$,

$$
\operatorname{Symb}\left(\Gamma_{0}(p) ; \kappa\right):=H_{c}^{1}\left(\Gamma_{0}(p) ; \mathcal{D}_{\kappa}\right) .
$$

Again, the standard Hecke operators can be viewed as acting naturally on the Banach space $H_{c}^{1}\left(\Gamma_{0}(p) ; \mathcal{D}_{\kappa}\right)$, but most importantly the Atkin-Lehner operator $U_{p}$ acts as a completely continuous operator, for any $p$-adic weight $\kappa$. 
The essential relationship between $p$-adic weights and 'classical' integral weights is given by the mapping, for $\kappa=k \in \mathbf{Z}$,

$$
\Phi: \mathcal{D}_{\kappa} \rightarrow P_{k}
$$

given as follows. Write $(X-x)^{k}:=\sum_{j=0^{k}} f_{j}(X) x^{j}$ as a polynomial in ('lower case') $x$, giving as coefficients, a sequence of polynomials, $f_{j}(X) \in P_{k} \subset A_{\kappa}$, in ('upper case') $X$. For $\mu \in \mathcal{D}_{\kappa}$ viewed as a linear functional on $\mathcal{A}$, form

$$
\mu_{j}:=\left\langle\mu, f_{j}(x)\right\rangle \in \mathbf{Q}_{p},
$$

and, finally, organize all these $\mu_{j}$ as coefficients of a polynomial in $X$ :

$$
\Phi(\mu):=\sum_{j=0}^{k} \mu_{j} X^{j} \in P_{k}
$$

giving our mapping

$$
\mu \mapsto \Phi(\mu)
$$

One shows that $\Phi$ is equivariant with respect to the actions of $\Gamma_{0}(p)$ and Hecke operators, and the all-important compact operator $U_{p}$, and therefore induces a correspondingly equivariant homomorphism of the modules of modular symbols:

$$
H_{c}^{1}\left(\Gamma_{0}(p) ; \mathcal{D}_{\kappa}\right) \stackrel{\Phi}{\longrightarrow} H_{c}^{1}\left(\Gamma_{0}(p) ; P_{k}\right)
$$

that respects slopes for $U_{p}$, i.e. if - for $h$ a non-negative real number-the superscript ${ }^{(h)}$ refers to the submodule of $U_{p}$-eigenvalue slope $\leq h$, we have the cut-offs of $\Phi$ for any slope, i.e., homomorphisms

$$
H_{c}^{1}\left(\Gamma_{0}(p) ; \mathcal{D}_{\kappa}\right)^{(h)} \stackrel{\Phi^{(h)}}{\longrightarrow} H_{c}^{1}\left(\Gamma_{0}(p) ; P_{k}\right)^{(h)}
$$

and Glenn's Comparison Theorem sometimes referred to as a Control Theorem which is an "overconvergent modular symbol analogue" of Robert Coleman's classical comparison theorem that relates $p$-adic overconvergent eigenforms of slope $<k+1$ to classical eigenforms ${ }^{14}$ :

Theorem 1. If $\kappa=k \geq 0$ and $h<k+1$ then $\Phi^{(h)}$ is an isomorphism:

$$
H_{c}^{1}\left(\Gamma_{0}(p) ; \mathcal{D}_{\kappa}\right)^{(h)} \stackrel{\Phi^{(h)}}{\simeq} H_{c}^{1}\left(\Gamma_{0}(p) ; P_{k}\right)^{(h)}
$$

\footnotetext{
${ }^{14}$ Coleman's Theorem, in turn, is a generalization of Hida's Theorem which proved the comparison for eigenforms of slope 0 .
} 
The fundamental importance of this interpolation, moving from the discrete family of cohomology with coefficients in symmetric power representations to continuous ( $p$-adically rigid analytic) families of cohomology with coefficients in the distribution modules defined by Glenn cannot be overestimated. What I have alluded to here - the initial definitions and theorems of this technology introduced by Glenn has led to a substantial amount of excellent mathematics, and will continue to do so.

Works continuing further in this direction include Glenn Steven's collaborative work with Avner Ash that moves from $\mathrm{GL}_{2}$ to to $\mathrm{GL}_{n}$ :

- $p$-adic deformations of arithmetic cohomology (February 2008 preprint)

- $p$-adic deformations of cohomology classes of subgroups of $\mathrm{GL}(n, \mathbf{Z})$, Journées Arithmétiques (Barcelona, 1995). Collect. Math. 48 (1997), no. 1-2, 1-30.

It includes his collaboration with Avner Ash and David Pollack:

- Rigidity of $p$-adic cohomology classes of congruence subgroups of $\mathrm{GL}(n, \mathbf{Z})$. Proc. Lond. Math. Soc. (3) 96 (2008), no. 2, 367-388

as well as his collaborative work with Rob Pollack:

- Overconvergent modular symbols and p-adic L-functions. Ann. Sci. Éc. Norm. Supér. (4) 44 (2011), no. 1, 1-42.

- Critical slope p-adic L-functions. J. Lond. Math. Soc. (2) 87 (2013), no. 2, 428-452.

which give a constructive proof of Theorem 1 and use that to establish polynomial-time algorithms for explicit computation of associated $p$-adic $L$-functions (even in the case of critical slope; i.e., when $h=k+1)$.

And his very recent work joint with Fabrizio Andreatta and Adrian Iovita which-among other things - establishes a $p$-adically varying Eichler-Shimura-type mapping.

Andreatta, Iovita, and Stevens, viewing $H_{c}^{1}\left(\Gamma_{0}(p) ; \mathcal{D}_{\kappa}\right)^{(h)}$ for appropriate ( $p$-adic) weights $\kappa$ as having a natural $G_{\mathbf{Q}_{p}}=\operatorname{Gal}\left(\overline{\mathbf{Q}}_{p}\right)$-action (via its relation to étale cohomology), establish what one might call an 'Eichler-Shimura relationship' for $H_{c}^{1}\left(\Gamma_{0}(p) ; \mathcal{D}_{\kappa}\right)^{(h)} \otimes \mathbf{C}_{p}(1)$, equivariant with respect to $G_{\mathbf{Q}_{p}}$ and Hecke-module structures. Moreover, this varies $p$-adically in the weight $\kappa$ (they must exclude some isolated weights from the $p$-adic parts of weight space over which they work). 


\section{Part V}

\section{The derivative of the $U_{p}$-eigenvalue with respect to weight}

There is not enough time in this hour to do more than mention the far-reaching idea of Ralph Greenberg and Glenn Stevens (for weight 2) in

- On the conjecture of Mazur, Tate, and Teitelbaum, p-adic monodromy and the Birch and Swinnerton-Dyer conjecture (Boston, MA, 1991), pp. 183-211 in Contemp. Math. 165 Amer. Math. Soc., Providence, RI, 1994,

- $p$-Adic L-functions and p-adic periods of modular forms. Invent. Math. 111 (1993), no. 2, 407-447.

and developed in various ways by Glenn for arbitrary even weights $k \geq 2$, e.g., in

- Coleman's $\mathcal{L}$-invariant and families of modular forms, Astérisque 331 (2010) 1-12.

This issue is to study the case when the order of vanishing at $s=k / 2$ of the $p$-adic $L$-function of a modular newform $f$ of even weight $k \geq 2$ is higher than the order of vanishing at the "same" point $s=1$ of the classical $L$-function. This phenomenon is known to happen when $f$ is of level $p m$ with $(p, m)=1$ and is split multiplicative at $p$; specifically, when the $U_{p}$-eigenvalue of the eignform $f$

is $p^{\frac{k-2}{2}}$. For example, consider the case where the classical $L$-function does not vanish at $s=k / 2$ and-deprived of its transcendental period-takes some value $A \in \mathbf{Q}^{*}$. The natural question, then, is the give an interpretation, and theoretical understanding (and an explicit computation) of the ratio

$$
\frac{L_{p}^{\prime}(E, k / 2)}{A} \in \mathbf{Q}_{p}
$$

where $L_{p}^{\prime}(E, k / 2):=\frac{d}{d s} L_{p}(E, s)_{\mid s=k / 2}$.

The answer that Ralph and Glenn give in the case $k=2$ (and that Glenn extends to general even weight) is striking. Viewing $f$ as a point on the appropriate eigencurve, the ratio $\frac{L_{p}^{\prime}(E, k / 2)}{A}$ is given, up to a simple factor, by the value - at the point $f$-of the derivative of the $U_{p}$-eigenvalue along the eigencurve with respect to the weight.

This opens up an important channel in our subject, and represents but one of the many contributions of Glenn Stevens to the expansion and enrichment of number theory. 


\section{References}

[1] Deninger, C.: How to recover an $L$-series from its values at almost all postive integers. Some remarks on a formula of Ramanujan, Proc. Indian Acad. Sci. Math. Sci. 110 (2000), no. 2, 121-132.

[2] Flach, M.: A finiteness theorem for the symmetric square of an elliptic curve Invent. Math. 109 (1992), no. 2, 307-327

[3] Kolyvagin, V.: Euler systems, pp. 435-483 in: The Grothendieck Festschrift (Vol. II), P. Cartier et al., eds., Prog. in Math 87, Boston: Birkhäuser (1990)

[4] Mazur, B.: Galois Deformations and Hecke Curves, http://www.math.harvard.edu/ mazur/ papers/scanGalois.pdf

[5] Rubin, K.: Euler systems and modular elliptic curves pp. 351-367 in: Galois representations in arithmetic algebraic geometry, A. J. Scholl and R. L. Taylor, eds., London Math. Soc. Lect. Notes 254 Cambridge: Cambridge Univ. Press (1998)

[6] Rubin, K.: Euler Systems Annals of Math. Studies 147, Princeton: Princeton University Press (2000)

[7] Scholl, A. J. An introduction to Kato's Euler systems, pp. 379-460 in Galois representations in arithmetic algebraic geometry (Durham, 1996), London Math. Soc. Lecture Note Ser., 254 Cambridge Univ. Press, Cambridge (1998)

[8] Serre, J.-P.: Formes modulaires et fonctions zêta p-adiques pp. 191-268 in Modular functions of one variable, III (Proc. Internat. Summer School, Univ. Antwerp, 1972), Lecture Notes in Math 350 Berlin, New York: Springer-Verlag (1973)

[9] Weston, T.: Algebraic cycles, modular forms and Euler systems J. Reine Angew. Math. 543 (2002), 103-145 